\title{
Repeated Exposure to Rewarding Brain Stimulation Downregulates GluR1 Expression in the Ventral Tegmental Area
}

\author{
William A. Carlezon, Jr., Ph.D., Mark S. Todtenkopf, M.S., Donna L. McPhie, Ph.D,. \\ Patricia Pimentel, B.S., Andrea M. Pliakas, B.S., James R. Stellar, Ph.D., and Monika Trzcinska, Ph.D.
}

There is considerable evidence that drug reward and brain stimulation reward (BSR) share common neural substrates. Although it is known that exposure to drugs of abuse causes a variety of molecular changes in brain reward systems, little is known about the molecular consequences of BSR. We report that repeated exposure to rewarding stimulation of the medial forebrain bundle (MFB) selectively decreases expression of GluR1 (an AMPA receptor subunit) in the $V T A$, without effect on expression of several other proteins (GluR2, NMDAR1, tyrosine hydroxylase). This effect of BSR on GluR1 expression is opposite of that caused by intermittent exposure to cocaine and morphine, which are known to elevate GluR1 expression in the VTA.

Considering that elevated GluR1 expression in the VTA has been associated with increased sensitivity to drug reward, the finding that BSR and drugs of abuse have opposite effects on GluR1 expression in this region may provide an explanation for why the reward-related effects of many drugs (cocaine, morphine, amphetamine, PCP, nicotine) do not sensitize with repeated testing in BSR procedures that quantify reward strength.

[Neuropsychopharmacology 25:234-241, 2001]

(C) 2001 American College of Neuropsychopharmacology.

Published by Elsevier Science Inc.
KEY WORDS: Brain stimulation reward; Lateral hypothalamus; Glutamate; AMPA; Dopamine; Protein; Addiction; Rat

Electrical brain stimulation, like intravenous administration of drugs of abuse, can establish arbitrary response habits (e.g., lever-pressing) in rats (see Wise 1996). Although there are many sites at which brain stimulation is rewarding, stimulation of the medial forebrain bundle (MFB) at the level of the lateral hypothalamus (LH) produces high response rates without

From the Department of Psychiatry, Harvard Medical School, McLean Hospital, Belmont, MA (WAC, DLM, AMP); and Department of Psychology, Northeastern University, Boston, MA (MST, PP, JRS, MT).

Address correspondence to: William A. Carlezon Jr., Ph.D., Department of Psychiatry, McLean Hospital, MRC 217, 115 Mill Street, Belmont MA 02478.

Received September 29, 2000; revised January 10, 2001; accepted January 23, 2001. evidence of aversive effects. Moreover, it is thought that the MFB is part of a final common path for the rewarding effects of brain stimulation at a variety of limbic sites (Wise 1996).

Many drugs of abuse-including morphine, cocaine, amphetamine, phencyclidine (PCP), and nicotine-potentiate the rewarding impact of MFB stimulation (see Wise 1996). When tested in the "curve-shift" variant of the brain stimulation reward (BSR) paradigm, these drugs increase the potency of the stimulation, causing leftward shifts in the functions that relate response strength to stimulation strength (Gallistel and Freyd 1987; Frank et al. 1988). Such shifts suggest additivity between the rewarding effects of the drug and the rewarding effects of the stimulation. Conversely, agents that attenuate drug reward (dopamine or opioid antagonists) attenuate BSR on their own, and block the ability of drugs of abuse to cause leftward shifts (Gallistel and Freyd 1987; Rompré and Wise 1989). Also, microinjec- 
tions of drugs of abuse potentiate BSR in the same brain regions in which they are rewarding in their own right (Wise 1996). Together, these findings are often considered evidence that drug reward and BSR share common neural substrates.

Repeated exposure to drugs of abuse can alter their rewarding effects in rats. Pre-exposure to amphetamine, cocaine, and morphine facilitates the ability of these drugs to establish conditioned place preferences (Lett 1989). Similarly, rats pre-exposed to amphetamine or cocaine learn more rapidly to self-administer these drugs intravenously (Piazza et al. 1990; Horger et al. 1990). These findings suggest that drug reward undergoes sensitization (reverse-tolerance) with repeated intermittent treatment. However, in curve-shift variants of the BSR paradigm, similar regimens do not produce any change in the strength of the reward-related effects of these drugs: there is no evidence of sensitization (or tolerance) to the rewarding effects of cocaine (Frank et al. 1988; Bauco and Wise 1997), amphetamine (Wise and Munn 1993), morphine (Bauco et al. 1993), PCP (Carlezon and Wise 1993), or nicotine (Bauco and Wise 1994) with repeated intermittent treatment. These findings raise the possibility that pre-exposure to electrical stimulation of the MFB during BSR training causes adaptations that prevent further changes in the rewarding strength of the drugs.

Drug exposure causes changes in gene expression within brain reward systems, some of which may contribute to these alterations in drug sensitivity. Stimulants and opiates elevate expression of the immediateearly gene $c$-fos throughout the striatum, including the nucleus accumbens (Graybiel et al. 1990; Curran et al. 1996; Badiani et al. 1998; Kelz et al. 1999). Morphine and cocaine elevate expression of tyrosine hydroxylase $(\mathrm{TH})$, the rate-limiting enzyme in dopamine synthesis, in the VTA (Beitner-Johnson and Nestler 1991).

Repeated intermittent exposure to these drugs also elevates expression of the AMPA receptor subunit GluR1 in the VTA (Fitzgerald et al. 1996; Churchill et al. 1999), which may explain increases in AMPA receptor function seen in dopamine neurons within this region after drug exposure (Zhang et al. 1997). Repeated cocaine elevates expression of the NMDA receptor subunit NMDAR1 in the VTA (Fitzgerald et al. 1996), and can elevate GluR1 in the nucleus accumbens (Churchill et al. 1999). In some cases, these changes in gene expression have been directly linked to changes in the motivational aspects of drugs of abuse. In the nucleus accumbens, expression of Fos-related proteins (i.e., $\Delta$ FosB) increases sensitivity to the rewarding effects of cocaine, whereas elevated GluR1 expression increases sensitivity to the aversive effects of cocaine (Kelz et al. 1999). In the rostral VTA, elevated GluR1 expression causes dramatic increases in the rewarding effects of morphine (Carlezon et al. 1997, 2000). Elevated GluR1 expression in the VTA might trigger a cascade of molecular changes that cause behavioral adaptations (see Self and Nestler 1995) because it favors the cellular influx of Ca2+ (Hollmann and Heinemann 1994; Seeberg et al. 1998), which plays an important role in many types of neuroadaptations (see Zucker 1999). Considering that sensitized behavioral responses can outlast elevated GluR1 expression in the VTA (Carlezon et al. 1997), it seems likely that this transient neuroadaptation contributes to the induction, rather than the expression, of sensitization. Indeed, there is considerable evidence that the induction of sensitization requires neuroadaptations in the VTA, whereas the expression of sensitization involves subsequent neuroadaptations in VTA target regions such as the nucleus accumbens (Kalivas and Stewart 1991; Wolf 1998).

Conversely, little is known about how BSR affects gene expression. Rewarding MFB stimulation elevates $c$-fos expression in various brain regions, including the VTA, nucleus accumbens, peduncopontine nucleus, locus coeruleus, septum, and bed nucleus of the stria terminalis (Arvanitogiannis et al. 1997; Flores et al. 1997; Hunt and McGregor 1998). In the VTA, c-fos expression does not appear to occur in TH-positive (dopaminergic) neurons (Hunt and McGregor 1998), and it desensitizes with repeated exposure to the stimulation (Nakahara et al. 1999). However, it is not known if these changes have behavioral relevance. Moreover, the effect of BSR on expression of other proteins with established behavioral relevance (i.e., GluR1 in the VTA) has not been studied.

The goal of the present study was to determine if the effects of repeated intermittent exposure to rewarding brain stimulation has the same effects on the expression of GluR1, GluR2, NMDAR1, and TH as does repeated intermittent exposure to cocaine and opiates. We report here that there are important differences in the molecular consequences of these two types of rewarding stimuli. The differences, particularly in the case of GluR1 expression, may explain why the rewarding effects of drugs of abuse do not sensitize with repeated testing in the BSR paradigm.

\section{MATERIALS AND METHODS}

Sixteen male Sprague-Dawley rats (350-450 g; Charles River Laboratories) were used. Rats were individually housed in standard plastic cages within a colony room on a reverse $12 \mathrm{hr}$ light: $12 \mathrm{hr}$ dark cycle (lights off at 7:00-19:00), with free access to food and water. Each rat was anesthetized with pentobarbital (50 mg/ $\mathrm{kg}$, i.p.), given atropine sulfate $(0.25 \mathrm{mg} / \mathrm{kg}$, s.c. $)$ to reduce bronchial secretions, and implanted with a monopolar, stainless steel electrode (Plastics One) aimed at the right MFB, at the level of the lateral hypothalamus $(3.0 \mathrm{~mm}$ posterior to bregma, $1.6 \mathrm{~mm}$ lateral from midsaggital 
suture, and $7.5 \mathrm{~mm}$ below dura) (Paxinos and Watson 1986; see Stellar et al. 1988). Skull screws (which served as the ground) and the electrode were secured to the skull with dental acrylic. All procedures were conducted in accordance with the NIH Guide for the Care and Use of Laboratory Animals.

One week after surgery, rats in the Experimental (E) group were trained on a continuous reinforcement schedule (FR1) to lever press for trains of $0.25 \mathrm{sec}$ square-wave monopolar cathodal pulses $(0.1 \mathrm{msec}$ pulse duration), at a set frequency of $100 \mathrm{~Hz}$. Stimulation was delivered by Stimtek stimulator-microcontrollers. For each rat, the minimal stimulation current (316$398 \mu \mathrm{A})$ that produced reliable responding with no signs of aversion (turning away from the lever, vocalization, and/or seizures) was used. All rats pressed reliably by the end of this $1 \mathrm{hr}$ training session. On each of the following nine days, rats were allowed to self-stimulate on an FR-1 schedule of reinforcement for $1 \mathrm{hr}$. Sessions began with illumination of a house light in the test chamber, and non-contingent delivery of a single train of priming stimulation. Control (C) animals were placed in the same operant chambers for $1 \mathrm{hr}$ each day for 10 consecutive days, but did not receive any stimulation. These procedures were conducted during the dark phase of the rats' light cycle.

To quantify the amount of stimulation that each rat received during each session, charge $(Q)$ was calculated according to a minor modification of the formula $Q=$ INd, where $Q=$ charge $(\mu \mathrm{C}), I=$ Current $(\mu \mathrm{A}), N=$ number of pulses in a $0.25 \mathrm{sec}$ train, and $d=$ pulse duration (0.0001 sec) (see Gallistel 1978). This formula combines all stimulation parameters into a single representative value for each stimulation site per subject. With this method, the excitability of the brain tissue under the tip of the electrode can be assessed and compared among many brain regions supporting BSR, as well as for the subregions within a specific area of interest (see Bielajew and Trzcinska 1998). Thus $Q$ is an index of the amount of rewarding stimulation that each rat receives. Usually, $Q$ has been used to reflect the characteristics of sites supporting BSR in mapping studies, where only a limited number of rate-frequency curves can be obtained because of performance deficits, seizures, etc. Since no rate-frequency curves were collected in the present study, $Q$ was calculated by multiplying current, pulse duration, number of pulses (which in a $0.25 \mathrm{sec}$ train was 25 for $100 \mathrm{~Hz}$ stimulation) and the number of reinforcements per day to obtain a final average value per subject.

Rats were killed by decapitation $24-26 \mathrm{hr}$ after the final brain stimulation (or control) session, and brains were placed immediately in ice-cold artificial cerebrospinal fluid (aCSF) buffer. A Stoelting tissue slicer was used to obtain $1.0 \mathrm{~mm}$-thick coronal slices of brain containing the rostral-central aspects of the VTA $(\sim 4.8-$ $\sim 5.8$ posterior to bregma), and a blunted 15 gauge needle was used to collect bilateral tissue punches. Tissue from each rat was homogenized in $250 \mu \mathrm{l}$ of $1 \%$ SDS, and protein content was determined by the method of Lowry et al. (1951). Tissue from each sample $(15 \mu \mathrm{g})$ was then subjected to one-dimensional SDS-polyacrylamide gel electrophoresis on $7.5 \%$ polyacrylamide resolving gels, and transferred electrophoretically onto polyvinylidene difluoride (Millipore) membranes for western immunoblot analysis (Western Star immunodetection system; Tropix) (see Carlezon et al. 1997).

Separate blots were conducted for each glutamate receptor subunit protein (GluR1: Chemicon, 1:1000; GluR2/3: Upstate Biotechnologies, Inc., 1:333; NMDAR1: Pharmingen, 1:1000), but TH (Chemicon, 1:1000) was analyzed on each membrane. The GluR2/3 antibody recognizes GluR2 almost exclusively, even though it is directed against a portion of the protein with some homology with GluR3 (see Prince et al. 1995). Equivalent protein loading was confirmed by amido black staining of the membranes. Levels of immunoreactivity were quantified using computer-assisted densitometry (MCID, Imaging Research Inc.), and between-group differences in relative optical density (signal minus background) for each protein were analyzed using Student's t-tests.

The portion of the brain anterior to the VTA (containing the MFB at the level of the LH) was placed in $4 \%$ paraformaldehyde solution. Sections $(40 \mu \mathrm{M})$ were stained with cresyl violet to confirm electrode placements.

\section{RESULTS}

One rat (C6) in the $C$ (non-stimulated) condition was euthanized before the completion of the experiment because its electrode became dislodged. No tissue was collected from this rat.

All rats in the E (stimulated) group rapidly learned to press, indicating that the brain stimulation was rewarding. The average daily $Q$ received by each rat was 2939.4 ( \pm 257.6 , S.E.M.) $\mu$ C (Table 1), which is well within the range of values obtained in previous studies (Bielajew and Trzcinska 1998). Electrode placements for each rat in the E group were located within the MFB at the level of the LH (Figure 1). Electrode placements for each rat in the $C$ group were similarly situated in the MFB, at sites that would likely support self-stimulation (this was impossible to test in the present experiment).

Repeated intermittent exposure to rewarding MFB stimulation $(1 \mathrm{hr} /$ day $\times 10$ days $)$ dramatically and selectively decreased GluR1 expression in the VTA $\left(\mathrm{t}_{13}=\right.$ $2.70, p<.02$ ) to $49 \%$ of control levels (Figure $2 \mathrm{~A}$ ). On the same blot on which GluR1 expression was significantly decreased, $\mathrm{TH}$ expression was unchanged $\left(\mathrm{t}_{13}=\right.$ $0.10, p=.91$, n.s.) (Figure 2B). Rewarding MFB stimula- 
Table 1. Average Daily Charge $(Q)$ for Each Rat Exposed to Rewarding MFB Stimulation for 10 Days, and Group Mean

\begin{tabular}{|c|c|c|c|c|c|c|c|c|c|}
\hline Subject & E1 & E2 & E3 & E4 & E5 & E6 & E7 & E8 & Mean \\
\hline $\mathrm{Q}(\mu \mathrm{C})$ & 3051.1 & 3731.6 & 2196.6 & 2190.7 & 3578.2 & 3788.6 & 2025.1 & 2955.2 & 2939.4 \\
\hline \pm SEM & 73.0 & 69.5 & 164.0 & 152.7 & 257.9 & 104.5 & 166.3 & 177.7 & 257.6 \\
\hline
\end{tabular}

tion did not alter GluR2 ( $\mathrm{t}_{13}=1.04, p=.37$, n.s.) (Figure $2 \mathrm{C})$ or NMDAR1 $\left(\mathrm{t}_{13}=0.60, p=.58\right.$, n.s.) expression (Figure 2D).

There was no correlation between $Q(\mu \mathrm{C})$ and levels of GluR1 expression in the VTA (relative optical density) $\left(\mathrm{F}_{1,6}=0.64, p=.45\right.$, n.s.) (Figure 3 ).

\section{DISCUSSION}

Repeated intermittent exposure to rewarding MFB stimulation decreases GluR1 expression in the VTA,

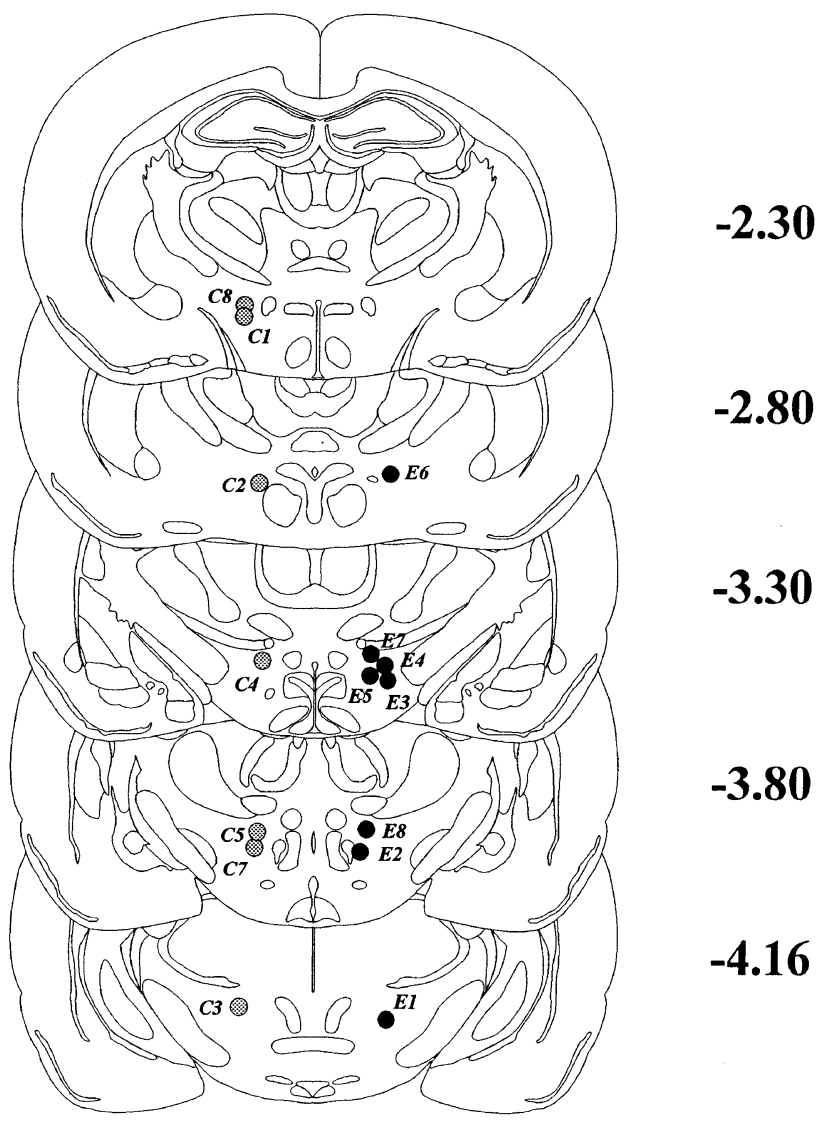

Figure 1. The locations of LH sites for the control (C) and experimental (E) and groups. Distance posterior to bregma $(\mathrm{mm})$ is indicated on the right. The circles (gray for $\mathrm{C}$ and black for E groups) localize the electrode tips, and the alphanumeric identifies the individual subjects. Although all electrodes were implanted in the right hemisphere, the groups are depicted separately for clarity (schematics from software [Neurographics-The Rat Brain] based on Paxinos and Watson 1986). without any effect on expression of GluR2, NMDAR1, or TH. This effect of BSR on GluR1 expression is opposite to that caused by intermittent exposure to cocaine and morphine, which is known to increase GluR1 expression in the VTA (Fitzgerald et al. 1996; Churchill et al. 1999). Thus, these two types of rewarding stimuli (drugs and BSR) cause fundamentally different neuroadaptations in the brain reward system despite the observations that they share common neural substrates (i.e., the mesocorticolimbic dopamine system) and they have common effects on behavior (e.g., both stimulate activity and establish lever-pressing). These data suggest that the neural mechanisms of drug reward and brain stimulation reward are at least partially non-overlapping (see Arvanitogiannis et al. 1997; Hunt and McGregor 1998).

The downregulation of GluR1 expression appears correlated only with whether the rats received any rewarding stimulation, rather than with the amount of rewarding stimulation that each rat received. If decreased GluR1 expression was a non-specific effect of the stimulation itself, it would be expected that higher amounts of stimulation received $(Q)$ would result in less GluR1 expression (optical density) in the VTA. This correlation was not demonstrated by the methods that we employed in the present studies. Because we used the minimal amount of stimulation that would support responding for each rat (see Methods), the rewarding effect of the stimulation was qualitatively similar for all of the rats, whereas the amount of stimulation received $(Q)$ differed among the rats.

The lack of correlation between the amount of MFB stimulation received and the downregulation of GluR1 in the VTA raises the possibility that, instead, we might find a stronger correlation between decreased GluR1 expression and the actual rewarding value of the brain stimulation if we used more quantitative measurements to define reward strength. This question would be best addressed in future studies using the curve-shift variant of the BSR paradigm, in which it is possible to define the actual rewarding value of the stimulation with great precision. In the curve-shift paradigm, the rats are trained extensively over a wide range of stimulation parameters, producing essentially a "dose-response" analysis of the rewarding efficacy of the brain stimulation (each stimulation parameter represents a "dose"). Protein expression in the VTA could be compared among groups of rats self-administering low, intermediate, and high "doses" of rewarding MFB stimulation. It is im- 

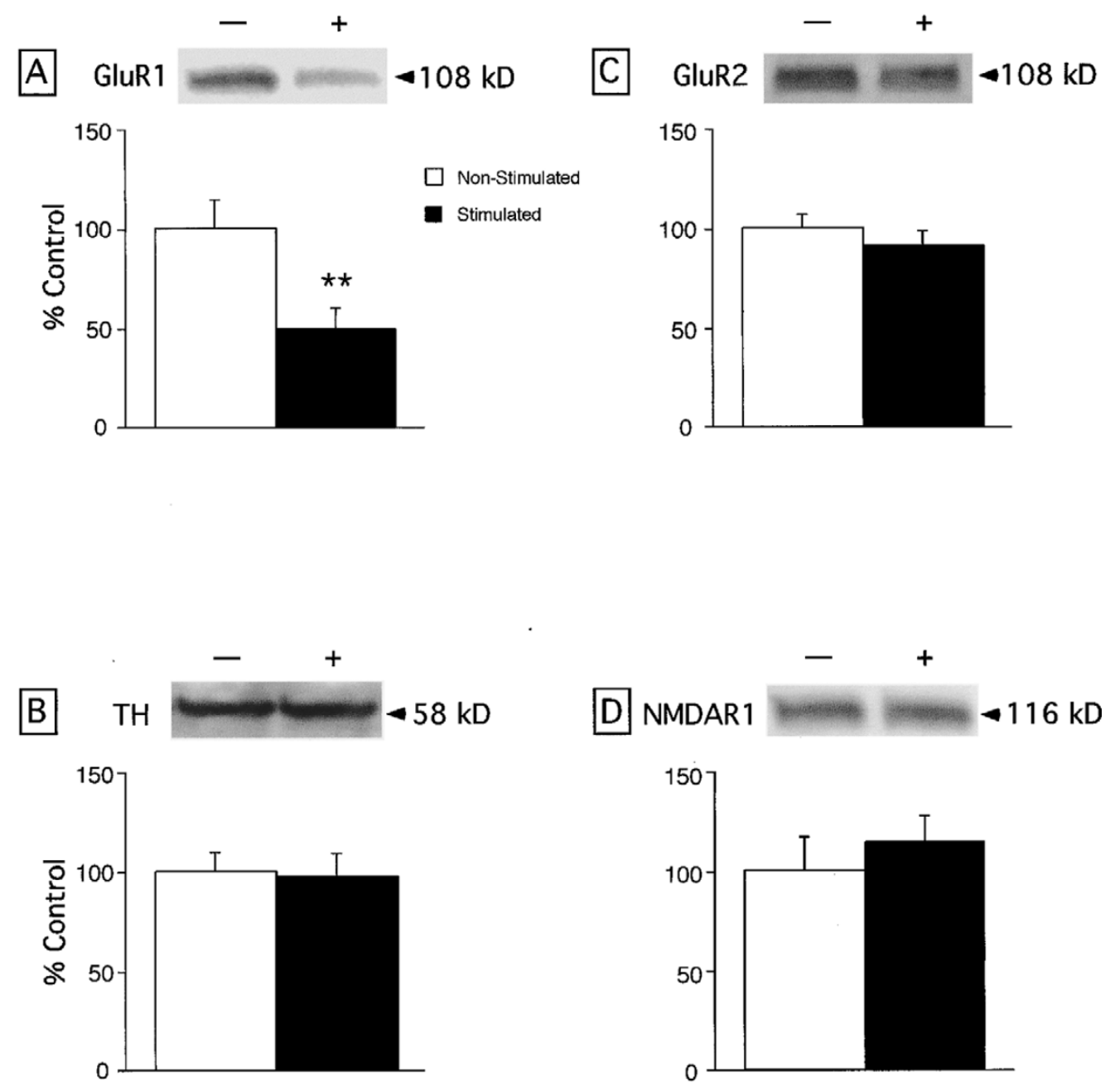

Figure 2. Protein immunoblots of VTA. Data are presented as percentage (mean \pm SEM) of protein expression in non-stimulated controls; - , non-stimulated; +, stimulated. Rewarding MFB stimulation decreases GluR1 expression (A) without affecting TH expression (B). The same two lanes from the same membrane are shown in (A) and (B). The stimulation did not alter expression of GluR2 (C) or NMDAR1 (D). ${ }^{* *} p<.02$, Student's t-test.

portant to note that the stimulation parameters that have low, intermediate, and high rewarding efficacy are different for each rat, and depend upon the exact placement of the electrode within the MFB; an amount of stimulation that supports maximal rates of responding in one rat may support only low rates in another rat. Therefore it is critical to show, as we have in the present studies, that protein expression in the VTA is not correlated merely with the amount of MFB stimulation received. Thus the present studies provide a rationale for performing more complex and labor-intensive studies involving the curve-shift paradigm.

Relative levels of GluR1 and GluR2 expression in the dopaminergic neuron-rich VTA are important because the subunit composition of AMPA receptors controls their function. High expression of GluR1 favors the formation of Ca2+-permeable (GluR1-homomeric) AMPA receptors (Hollmann and Heinemann 1994; Carlezon et al. 1997; Seeberg et al. 1998), which presumably increase sensitivity to the excitatory (depolarizing) effects of glutamate (see Neve et al. 1997). Conversely, high expression of GluR2 favors the formation of Ca2+-impermeable (hetereomeric) AMPA receptors, since this subunit contains a motif that blocks $\mathrm{Ca} 2+$ conductance (Hollmann and Heinemann 1994).

Repeated exposure to drugs of abuse selectively elevates GluR1 expression in the VTA (Fitzgerald et al. 1996; Churchill et al. 1999), and this change may explain, at least in part, the sensitized electrophysiological responses of VTA dopamine neurons seen in drugexposed rats (Zhang et al. 1997). Furthermore, selective elevations in GluR1 expression in the rostral VTA (the predominant region probed in the present studies) by viral-mediated gene transfer can increase responsivity to the rewarding effects of morphine (Carlezon et al. 1997, 2000), establishing causal relations between ele- 


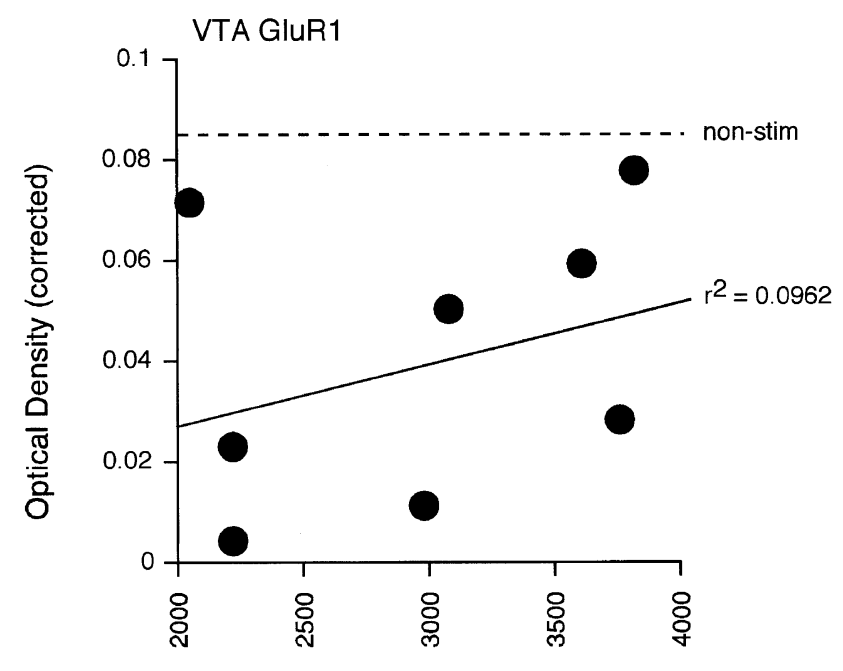

Avg. Daily Charge $(\mu \mathrm{C})$

Figure 3. Lack of correlation between the daily amount of charge $(Q)$ (mean \pm SEM) received by each rat and VTA levels of GluR1 expression (mean optical density \pm SEM) for each rat in the stimulated group. Relative optical density was calculated for each subject by subtracting background from signal, and $Q$ was obtained from Table 1 . Dashed line represents mean optical density of non-stimulated group.

vated GluR1 expression in this region and sensitization. Drug-related elevations in GluR1 expression in the VTA, a region known to be involved in the induction of sensitization (Kalivas and Stewart 1991; Wolf, 1998), may themselves be sufficient to explain sensitization (Carlezon et al. 1997; Zhang et al. 1997), or they may lead to Ca2+-dependent adaptations (see Self and Nestler 1995) in the VTA or other regions that also contribute to changes in drug sensitivity (Kalivas and Stewart 1991; Wolf 1998).

The present data strengthen the hypothesized association between elevated GluR1 expression in the VTA and sensitization of reward. Decreased GluR1 expression without changes in GluR2 expression would be expected to favor formation of GluR2-containing, Ca2+impermeable AMPA receptors in the VTA. Considering that increased GluR1 and/or Ca2+ flux in the VTA seems critical for the induction of sensitized drug responses (Carlezon et al. 1997; see also Wolf 1998), our data may provide an explanation for why repeated intermittent administration of drugs of abuse do not produce sensitization when tested in variants of the BSR paradigm that are sensitive to changes in reward strength (e.g., the "curve-shift" variant).

Acute administration of most drugs of abuse potentiates the rewarding strength of MFB stimulation (see Wise 1996). This effect indicates additivity between the rewarding effects of the stimulation and the rewarding effects of the drugs, and suggests that the drugs themselves are rewarding. However, the magnitude of the acute reward-related effects of cocaine (Frank et al. 1988; Bauco and Wise 1997), amphetamine (Wise and Munn 1993), morphine (Bauco and Wise 1993), PCP (Carlezon and Wise 1993), and nicotine (Bauco et al. 1994) does not change progressively with repeated intermittent drug treatment in the BSR paradigm. [There is a report in which brain stimulation pre-sensitizes rats to the locomotor-stimulating effects of amphetamine (Ben-Shahar and Ettenberg 1994), but this work involved direct rather than transynaptic stimulation of the VTA, which might result in different neuroadaptations.]

The lack of sensitization to drug reward in BSR procedures that use MFB stimulation is in stark contrast to findings in place conditioning and intravenous drug self-administration paradigms (Lett 1989; Piazza et al. 1990; Horger et al. 1990), which indicate that drug reward undergoes sensitization as the result of repeated exposure. This inconsistency among reward paradigms raises the possibility that pre-exposure to electrical stimulation of the MFB during BSR training causes adaptations that prevent further changes in the rewarding strength of the drugs. Our present data suggest that MFB stimulation causes a change (decreased GluR1 expression in the VTA) exactly opposite to that necessary for the induction and/or expression of sensitized responses to the rewarding effects of drugs of abuse (elevated GluR1 expression). Thus when given in combination with MFB stimulation, repeated intermittent drug administration may never result in the elevations in VTA GluR1 expression that are required to trigger sensitized responses to the rewarding effects of the drugs. This interaction can be addressed in future studies in which GluR1 expression is analyzed in the VTA of rats that receive drugs and rewarding MFB stimulation concurrently.

The molecular explanation for differences between the effects of rewarding MFB stimulation and drugs of abuse on GluR1 expression in the VTA is unknown. One important distinction between drugs and BSR is that drugs have pharmacological actions at receptor and/or transport proteins within brain reward circuitry, whereas the effects of BSR depend upon the electrically-stimulated release of endogenous transmitters. This difference would appear to be of little relevance, however, since stress (like drugs of abuse) also increases GluR1 expression in the VTA via release of endogenous transmitters (Fitzgerald et al. 1996). Regardless, the ability of rewarding MFB stimulation to decrease GluR1 expression selectively-at least among the proteins probed-in the VTA may offer a valuable method with which to address directly the role of VTA GluR1 in the behavioral, molecular, and electrophysiological correlates of sensitization. There are currently no described methods, other than antisense oligonucle- 
otides injected directly into the VTA, with which to selectively decrease GluR1 expression in this region. Conceivably, the ability of rewarding MFB stimulation to decrease GluR1 expression in the VTA may ultimately provide a working molecular model of protection against sensitization to the incentive-motivational properties of drugs and, by extension (see Robinson and Berridge 1993), resistance to addiction.

\section{ACKNOWLEDGMENTS}

Funded by grants to W.C. from National Alliance for Research on Schizophrenia and Depression (NARSAD) and the Nancy Lurie Marks Family Foundation. We thank A. Arvanitogiannis and anonymous reviewers for helpful comments.

\section{REFERENCES}

Arvanitogiannis A, Flores C, Shizgal P (1997): Fos-like immunoreactivity in the caudal diencephalon and brainstem following lateral hypothalamic self-stimulation. Behav Brain Res 88:275-279

Badiani A, Oates MM, Day HE, Watson SJ, Akil H, Robinson TE (1998): Amphetamine-induced behavior, dopamine release, and c-fos mRNA expression: modulation by environmental novelty. J Neurosci 18:10579-10593

Bauco P, Wang Y, Wise RA (1993): Lack of sensitization or tolerance to the facilitating effect of ventral tegmental area morphine on lateral hypothalamic brain stimulation reward. Brain Res 617:303-308

Bauco P, Wise RA (1994): Potentiation of lateral hypothalamic and midline mesencephalic brain stimulation reinforcement by nicotine: Examination of repeated treatment. J Pharmacol Exper Ther 271:294-301

Bauco P, Wise RA (1997): Synergistic effects of cocaine with lateral hypothalamic brain stimulation reward: Lack of tolerance or sensitization. J Pharmacol Exper Ther 283: 1160-1167

Beitner-Johnson D, Nestler EJ (1991): Morphine and cocaine exert common chronic actions on tyrosine hydroxylase in dopaminergic brain reward regions. J Neurochem 157:344-347

Ben-Shahar O, Ettenberg A (1994): Repeated stimulation of the ventral tegmental area sensitizes the hyperlocomotor response to amphetamine. Pharmacol Biochem Behav 48:1005

Bielajew C, Trzcinska M (1998) Activation of reward-relevant neurons in the caudate-putamen influences the development of medial prefrontal cortex self-stimulation: A moveable electrode mapping study. Act Neurobiol Exp 58:189-198

Carlezon WA Jr, Boundy VA, Haile CN, Lane SB, Kalb RG, Neve RL, Nestler EJ (1997): Sensitization to morphine induced by viral-mediated gene transfer. Science 277:812-814

Carlezon WA Jr, Haile CN, Coopersmith R, Hayashi Y, Malinow R, Neve RL, Nestler EJ (2000): Distinct sites of opi- ate reward and aversion within the midbrain identified using a herpes simplex virus vector expressing GluR1. J Neurosci 20(1-5):RC62

Carlezon WA Jr, Wise RA (1993): Phencyclidine-induced potentiation of brain stimulation reward: Acute effects are not altered by repeated administration. Psychopharmacology 111:402-408

Churchill L, Swanson CJ, Urbina M, Kalivas PW (1999): Repeated cocaine alters glutamate receptor subunit levels in the nucleus accumbens and ventral tegmental area of rats that develop behavioral sensitization. J Neurochem 72:2397-2403

Curran EJ, Akil H, Watson SJ (1996): Psychomotor stimulant- and opiate-induced c-fos mRNA expression patterns in the rat forebrain: Comparisons between acute drug treatment and a drug challenge in sensitized animals. Neurochem Res 21:1425-1435

Fitzgerald LW, Ortiz J, Hamedani AG, Nestler EJ (1996): Drugs of abuse and stress increase the expression of GluR1 and NMDAR1 glutamate receptor subunits in the rat ventral tegmental area: Common adaptations among cross-sensitizing agents. J Neurosci 16:274-282

Flores C, Arvanitogiannis A, Shizgal P (1997): Fos-like immunoreactivity in forebrain regions following selfstimulation of the lateral hypothalamus and the ventral tegmental area. Behav Brain Res 87:239-251

Frank RA, Martz S, Pommering T (1988): The effect of chronic cocaine on self-stimulation train-duration thresholds. Pharmacol Biochem Behav 29:755-758

Gallistel CR (1978): Self-stimulation in the rat: Quantitative characteristics of the reward pathway. J Comp Physiol Psychol 92:977-998

Gallistel CR, Freyd G (1987): Quantitative determination of the effects of catecholaminergic agonists and antagonists on the rewarding efficacy of brain stimulation. Pharmacol Biochem Behav 26:731-741

Graybiel AM, Moratalla R, Robertson HA (1990): Amphetamine and cocaine induce drug-specific activation of the c-fos gene in striosome-matrix compartments and limbic subdivisions of the striatum. Proc Natl Acad Sci U S A 87:6912-6916

Hollmann M, Heinemann S (1994): Cloned glutamate receptors. Ann Rev Neurosci 17:31-108

Horger BA, Shelton K, Schenk S (1990): Preexposure sensitizes rats to the rewarding effects of cocaine. Pharmacol Biochem Behav 37:707-711

Hunt GE, McGregor IS (1998): Rewarding brain stimulation induces only sparse Fos-like immunoreactivity in dopaminergic neurons. Neurosci 83:501-515

Kalivas PW, Stewart J (1991): Dopamine transmission in the initiation and expression of drug- and stress-induced sensitization of motor activity. Brain Res Rev 16:223244

Kelz MB, Chen JS, Carlezon WAJ, Whisler K, Gilden L, Beckmann A, Steffan C, Zheng YJ, Marotti L, Self DW, Tkatch T, Baranauskas G, Surmeier DJ, Neve RL, Duman RS, Piciotto MR, Nestler EJ (1999): Expression of the transcription factor $\mathrm{dFosB}$ in the brain controls sensitivity to cocaine. Nature 401:272-276

Lett BT (1989): Repeated exposures intensify rather than 
diminish the rewarding effects of amphetamine, morphine, and cocaine. Psychopharmacology 98:357-362

Lowry OH, Rosenbrough NJ, Farr AL, Randall RJ (1951): Protein measurement with the Folin phenol reagent. J Biol Chem 193:265-275

Nakahara D, Ishida Y, Nakamura M, Kuwahara I, Todaka K, Nishimori T (1999): Regional differences in desensitization of c-Fos expression following repeated self-stimulation of the medial forebrain bundle in the rat. Neuroscience 90:1013-1020

Neve RL, Howe JR, Hong S, Kalb RG (1997): Introduction of the glutamate receptor subunit 1 into motor neurons in vitro and in vivo using recombinant herpes simplex virus. Neuroscience 79:435-447

Paxinos G, Watson C (1986): The rat brain in stereotaxic coordinates. Sydney, Australia, Academic Press

Piazza PV, Deminiere JM, le Moal M, Simon H (1990): Stressand pharmacologically-induced behavioral sensitization increases vulnerability to acquisition of amphetamine self-administration. Brain Res 514:22-26

Prince HK, Conn PJ, Blackstone CD, Huganir RL, Levey AI (1995): Down-regulation of AMPA receptor subunit GluR2 in amygdaloid kindling. J Neurochem 64:462-465

Robinson TE, Berridge KC (1993): The neural basis of drug craving: An incentive-sensitization theory of addiction. Brain Res Rev 18:247-292

Rompré P-P, Wise RA (1989): Opioid-neuroleptic interaction in brainstem self-stimulation. Brain Res 477:144-151
Seeberg PH, Higuchi M, Sprengel R (1998): RNA editing of brain glutamate receptor channels: Mechanism and physiology. Brain Res Rev 26:217-229

Self DW, Nestler EJ (1995): Molecular mechanisms of drug reinforcement and addiction. Ann Rev Neurosci 18:463495

Stellar JR, Waraczynski M, Bruno JP (1988): Neonatal dopamine depletions spare lateral hypothalamic stimulation reward in adult rats. Pharm Biochem Behav 30: 365-370

Wise RA (1996): Addictive drugs and brain stimulation reward. Ann Rev Neurosci 19:319-340

Wise RA, Munn E (1993): Effects of repeated amphetamine injections on lateral hypothalamic brain stimulation reward and subsequent locomotion. Behav Brain Res 55:195-201

Wolf ME (1998): The role of excitatory amino acids in behavioral sensitization to psychomotor stimulants. Progr Neurobiol 54:679-720

Zhang Z-F, Hu X-T, White FJ, Wolf ME (1997): Increased responsiveness of ventral tegmental area dopamine neurons to glutamate after repeated administration of cocaine or amphetamine is transient and selectively involves AMPA receptors. J Pharmacol Exper Ther 281:699-706

Zucker RS (1999): Calcium- and activity-dependent synaptic plasticity. Curr Opin Neurobiol 9:305-313 